\title{
Thermal D mesons from anisotropic lattice QCD
}

\author{
Aoife Kelly ${ }^{1}$ and Jon-Ivar Skullerud ${ }^{1, a}$ \\ ${ }^{1}$ Department of Mathematical Physics, National University of Ireland Maynooth, Maynooth, Co Kildare, Ire- \\ land
}

\begin{abstract}
We present results for correlators and spectral functions of open charm mesons using $2+1$ flavours of clover fermions on anisotropic lattices. The D mesons are found to dissociate close to the deconfinement crossover temperature $T_{c}$. Our preliminary results suggest a shift in the thermal D meson mass below $T_{c}$. Mesons containing strange quarks exhibit smaller thermal modifications than those containing light quarks.
\end{abstract}

\section{Introduction}

The study of heavy quarks, and in particular charm quarks, in high temperature QCD has a long history. Until recently, however, the focus has been almost exclusively on quarkonia, ie the $c \bar{c}$ and $b \bar{b}$ systems. This has changed in the past few years, with an increased experimental and theoretical interest in open charm, including D meson flow [1] and yields [2-4].

There are several reasons why it is important to study open charm alongside charmonium. Since charm quarks are not created or destroyed thermally to any significant degree at the temperatures reached at RHIC and LHC, any decreased yield of charmonium states must be associated with an increased yield in open charm (although this can be hard to identify experimentally). It has also been suggested that the double ratio $R_{A A}(J / \psi) / R_{A A}(D)$ may be a better measure of medium modifications than the traditional $R_{A A}(J / \psi)$ as a number of systematic effects including cold nuclear matter effects cancel out in this ratio.

An important issue to understand in this context is to what extent open charm mesons experience thermal modifications below $T_{c}$, and whether some bound states may survive above $T_{c}$. It has for example been suggested that the abundance of $D_{s}$ states may increase relative to the states containing light quarks.

There have been very few lattice studies of open charm at high temperature so far [5-7]. These have used spatial correlators and cumulants to assess the possible survival of open charm bound states above $T_{c}$. Here we will for the first time present results for temporal correlators and spectral functions of open charm mesons.

\section{Methods}

This study uses the FASTSUM collaboration ensemble [8, 9], with 2+1 flavours of anisotropic clover fermions and a mean-field improved anisotropic Symanzik gauge action. The spatial lattice spacing

\footnotetext{
ae-mail: jonivar@thphys.nuim.ie
} 
Table 1. Lattice volumes $N_{s} \times N_{\tau}$, temperatures $T$ and number of configurations $N_{\text {cfg }}$ used in this work. The pseudocritical temperature $T_{c}$ was determined from the inflection point of the Polyakov loop [9].

\begin{tabular}{|ccccr|}
\hline$N_{s}$ & $N_{\tau}$ & $T(\mathrm{MeV})$ & $T / T_{c}$ & $N_{\text {cfg }}$ \\
\hline 24 & 128 & 44 & 0.24 & 500 \\
24 & 40 & 141 & 0.76 & 500 \\
24 & 36 & 156 & 0.84 & 500 \\
24 & 32 & 176 & 0.95 & 1000 \\
24 & 28 & 201 & 1.09 & 1000 \\
24 & 24 & 235 & 1.27 & 1000 \\
24 & 20 & 281 & 1.52 & 576 \\
24 & 16 & 352 & 1.90 & 1000 \\
\hline
\end{tabular}

is $a_{s}=0.123 \mathrm{fm}$ and the anisotropy $\xi=a_{s} / a_{\tau}=3.5$. The strange quark mass is tuned to its physical value, while the light quarks correspond to a pion mass $m_{\pi} \approx 380 \mathrm{MeV}$. Further details about the ensembles are given in table 1 . The action is identical to that used by the Hadron Spectrum Collaboration [10], and the zero temperature $\left(N_{\tau}=128\right)$ configurations were kindly provided by them. For the charm quarks, we have also used the parameters from the Hadron Spectrum Collaboration [11]. Both the configurations and the correlators were generated using the Chroma software package [12].

Information about hadronic states (including energies, widths and thresholds) in the medium is contained in the spectral function $\rho(\omega ; T)$, which for mesonic states is related to the euclidean correlator $G(\tau ; T)$ according to

$$
G(\tau ; T)=\int \rho(\omega ; T) K(\tau, \omega ; T) d \omega, \quad K(\tau, \omega ; T)=\frac{\cosh [\omega(\tau-1 / 2 T)]}{\sinh (\omega / 2 T)},
$$

Determining $\rho(\omega)$ from a given (noisy) $G(\tau)$ cannot be done exactly; we will here employ the maximum entropy method (MEM) to obtain the most likely spectral function for the given data. The spectral function is written in terms of a default model $m(\omega)$, which encodes prior information, and a set of $N_{b}$ basis functions $u_{k}(\omega)$ as

$$
\rho(\omega)=m(\omega) \exp \left[\sum_{k=1}^{N_{b}} b_{k} u_{k}(\omega)\right] .
$$

The standard implementation of MEM employs the singular value decomposition (SVD) of the kernel $K$,

$$
K\left(\omega_{i}, \tau_{j}\right)=K_{i j}=(U \Xi V)_{j i},
$$

and the basis functions $u_{k}$ are chosen to be the column vectors of $U$ corresponding to the $N_{s} \leq N_{\text {data }}$ singular values in the diagonal matrix $\Xi$. We will instead use a Fourier basis [13] which was found to yield more reliable results for the data at hand. The results have been cross-checked using the standard SVD basis and the SVD basis with extended search space [14], and found to be consistent within the uncertainties inherent in the respective methods.

The systematic uncertainty of the MEM can be avoided by studying the reconstructed correlator, defined as

$$
G_{r}\left(\tau ; T, T_{r}\right)=\int_{0}^{\infty} \rho\left(\omega ; T_{r}\right) K(\tau, \omega, T) d \omega,
$$

where $T_{r}$ is a reference temperature where the spectral function can be reliably constructed, usually chosen to be the lowest available temperature. It is clear that if there are no medium modifications, ie $\rho(\omega ; T)=\rho\left(\omega ; T_{r}\right)$ then $G_{r}\left(\tau ; T, T_{r}\right)=G(\tau ; T)$ (although the converse is not necessarily the case). 
The reconstructed correlator can also be computed directly from the underlying correlator $G\left(\tau ; T_{r}\right)$ without having to extract any spectral functions [15]. Using

$$
\frac{\cosh [\omega(\tau-N / 2)]}{\sinh (\omega N / 2)}=\sum_{n=0}^{m-1} \frac{\cosh [\omega(\tau+n N+m N / 2)]}{\sinh (\omega m N / 2)}
$$

where

$$
T=\frac{1}{a_{\tau} N}, T_{r}=\frac{1}{a_{\tau} N_{r}}, \quad \frac{N_{r}}{N}=m \in \mathbb{N}
$$

we find

$$
G_{r}\left(\tau ; T, T_{r}\right)=\sum_{n=0}^{m-1} G\left(\tau+n N, T_{r}\right) .
$$

In this study we will use $T_{r}=44 \mathrm{MeV}\left(N_{\tau}=128\right)$ as the reference temperature throughout, and will employ (7) to compute the reconstructed correlator, padding $G\left(\tau ; T_{r}\right)$ with zeros in the middle where necessary.

\section{Results}

We have computed correlators and spectral functions in the pseudoscalar and vector channels for light-charm $\left(D, D^{*}\right)$ and strange-charm $\left(D_{s}, D_{s}^{*}\right)$ mesons. Figure 1 shows the spectral functions at our lowest temperature in all four channels. We also show the ground and first excited states computed

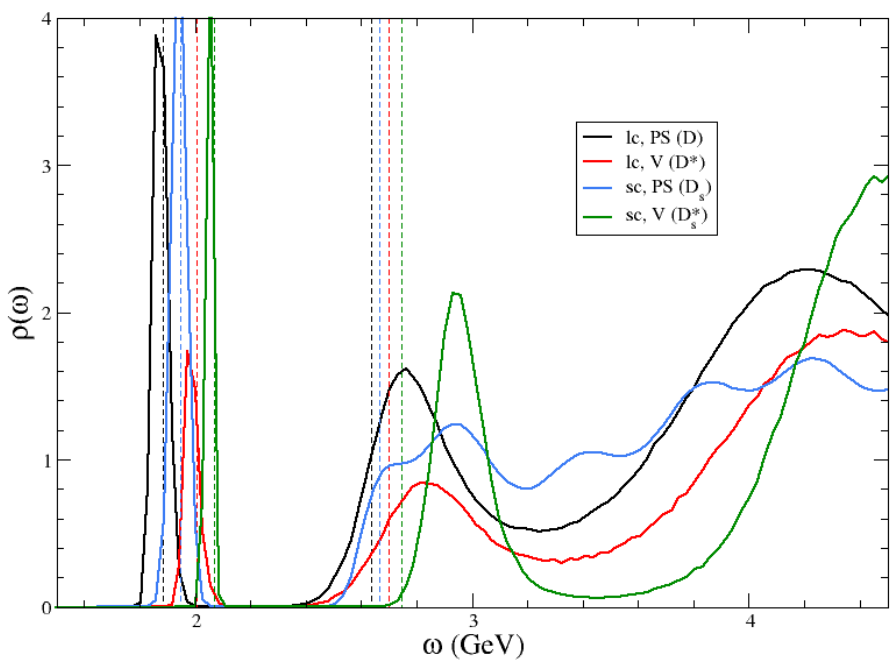

Figure 1. Zero temperature spectral function $\rho(\omega)$ in all four channels. The vertical lines show the ground states and first excited states computed by the HadSpec Collaboration [16].

by the Hadron Spectrum Collaboration [16] using a variational analysis. Comparing the two, we see that the ground state is very well reproduced by the MEM, while we are not able to reproduce the first excited state accurately with our current data. The wiggles seen in the $D_{s}$ spectral function are an artefact of the Fourier basis. 


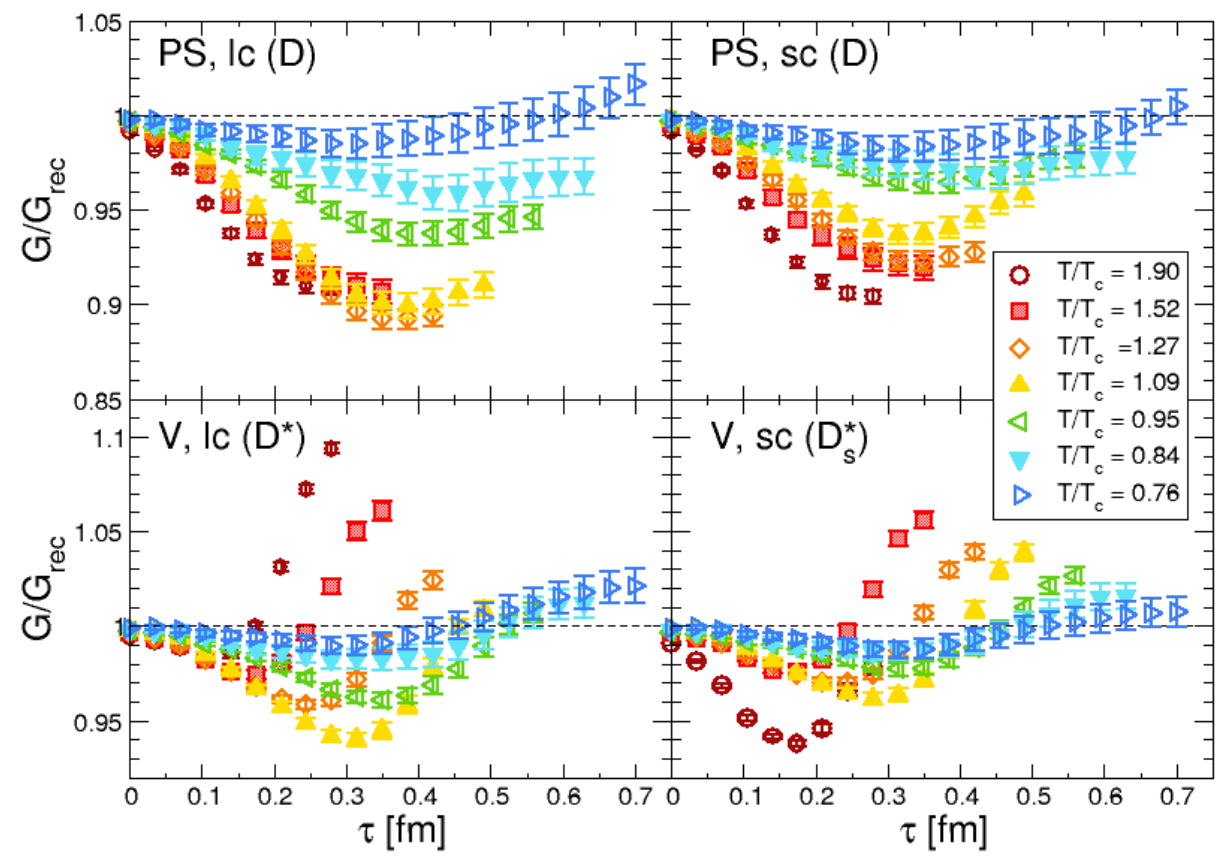

Figure 2. Open charm correlators $G(\tau ; T)$ divided by the reconstructed correlator $G_{r}\left(\tau ; T, T_{r}\right)$ computed from eq. (7) with $T_{r}=0.24 T_{c}$.

In figure 2 we show the correlators $G(\tau)$ divided by the reconstructed correlators $G_{r}(\tau)$ at the same temperature, for all four channels. This ratio should be 1 if there are no thermal modifications. We observe that the correlator at $T=0.76 T_{c}$ is consistent with no modifications in all channels, but there are thermal modifications already at $T=0.84 T_{c}$, in particular in the $D$ meson channel. Above $T_{c}$, the modifications become significant in all channels. We also see that except for the highest temperature $\left(T=1.9 T_{c}\right)$, the modifications in the strange-charm sector are smaller than those in the light-charm sector, which may lend support to the hypothesis that $D_{s}$ yields may be increased relative to $D$ yields.

We now turn to the spectral functions, which are shown in figures 3 and 4 for the pseudoscalar and vector channels respectively. We see no sign of any surviving bound state above $T_{c}$, suggesting that all open charm states dissociate near $T_{c}$. It may appear that the dissociation occurs already at $T=0.95 T_{c}$, but it should be noted that the transition to the quark-gluon plasma is a broad crossover for our parameters, and that $T=0.95 T_{c}$ lies within the crossover region [9].

Interestingly, there are suggestions, in particular in the vector channel, of a thermal mass shift below $T_{c}$, with the ground state peak position at $T=0.76 T_{c}$ being considerably higher than at zero temperature. So far, this feature appears to be robust with respect to variations in the basis functions and default model, but work is still in progress to confirm this.

As will be apparent from fig. 2, the statistical uncertainties in our correlators are relatively large, at $O(1 \%)$. Typically, permille errors or smaller are required to unambiguously identify spectral features. The fairly broad features seen in figs 3 and 4 for $T \gtrsim T_{c}$ may hence be a reflection of our limited precision. We are currently working on improving on this by using multiple sources per configuration for our correlators. 

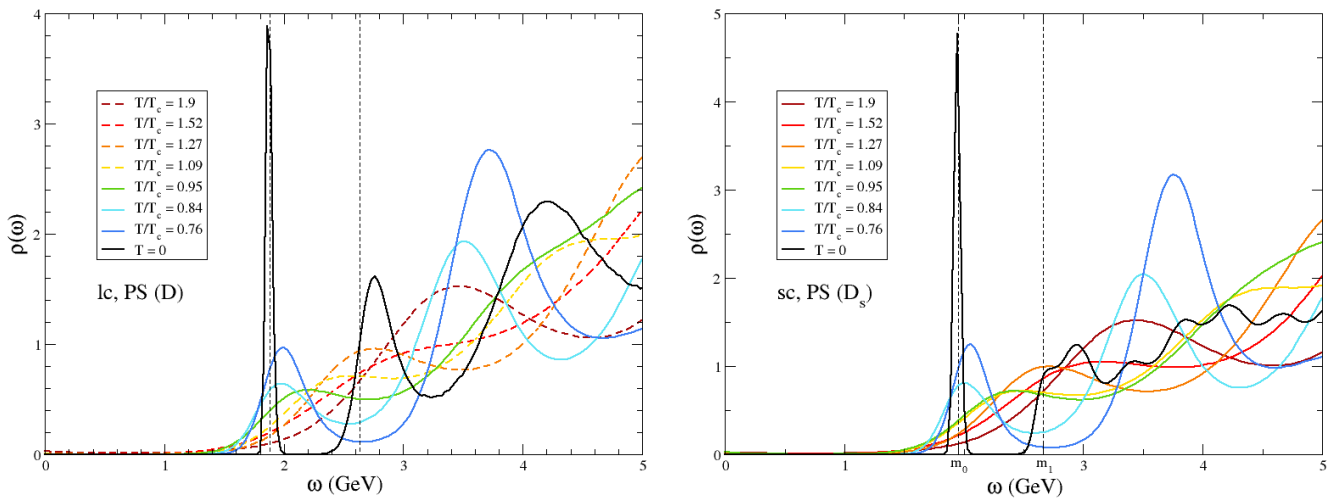

Figure 3. Pseudoscalar channel spectral function $\rho(\omega)$ for light-charm (left) and strange-charm (right) mesons. The vertical lines denote the ground and first excited state masses from ref. [16].
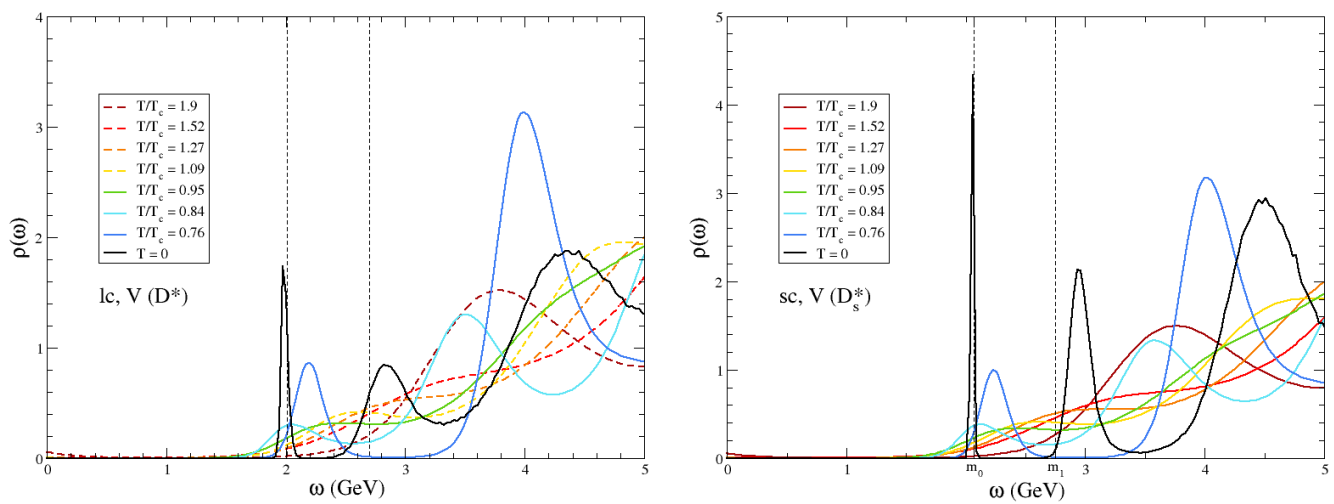

Figure 4. Vector channel spectral function $\rho(\omega)$ for light-charm (left) and strange-charm (right) mesons. The vertical lines denote the ground and first excited state masses from ref. [16].

\section{Summary and outlook}

We have carried out the first lattice calculation of temporal correlators and spectral functions of open charm mesons, both above and below the deconfinement crossover. We find clear evidence of thermal modifications already below the crossover, which may include a thermal mass shift. Above the crossover, we find no evidence of any surviving bound states. The thermal modifications are smaller for $D_{s}$ and $D_{s}^{*}$ mesons than for $D$ and $D^{*}$ mesons, which may point to an enhanced yield of $D_{s}$ relative to $D$ mesons in heavy ion collisions, in line with theoretical predictions and emerging experimental evidence [4].

We are currently working on improving our statistics using multiple sources, which would enable a more precise and reliable extraction of spectral functions. We are also carrying out a complete study of MEM systematics; so far, our main results do not appear to be affected by these systematics. We are also planning to cross-check the MEM results using alternative methods such as the BR method [17]. 


\section{Acknowledgments}

We thank Alexander Rothkopf for providing access to his MEM code and for numerous invaluable discussions. We also thank Gert Aarts and Chris Allton for fruitful discussions. We acknowledge the use of computational resources provided by ICHEC and the STFC funded DiRAC facility.

\section{References}

[1] B. Abelev et al. (ALICE), Phys. Rev. Lett. 111, 102301 (2013), 1305. 2707

[2] L. Adamczyk et al. (STAR), Phys. Rev. Lett. 113, 142301 (2014), 1404.6185

[3] J. Adam et al. (ALICE), JHEP 03, 081 (2016), 1509.06888

[4] J. Adam et al. (ALICE), JHEP 03, 082 (2016), 1509. 07287

[5] A. Bazavov, H.T. Ding, P. Hegde, O. Kaczmarek, F. Karsch et al., Phys.Lett. B737, 210 (2014), 1404.4043

[6] A. Bazavov, F. Karsch, Y. Maezawa, S. Mukherjee, P. Petreczky, Phys. Rev. D91, 054503 (2015), 1411.3018

[7] S. Mukherjee, P. Petreczky, S. Sharma, Phys. Rev. D93, 014502 (2016), 1509.08887

[8] G. Aarts, C. Allton, T. Harris, S. Kim, M.P. Lombardo et al., JHEP 1407, 097 (2014), 1402.6210

[9] G. Aarts, C. Allton, A. Amato, P. Giudice, S. Hands, J.I. Skullerud, JHEP 1502, 186 (2015), 1412.6411

[10] R.G. Edwards, B. Joó, H.W. Lin, Phys.Rev. D78, 054501 (2008), 0803.3960

[11] L. Liu, G. Moir, M. Peardon, S.M. Ryan, C.E. Thomas, P. Vilaseca, J.J. Dudek, R.G. Edwards, B. Joó, D.G. Richards (Hadron Spectrum), JHEP 07, 126 (2012), 1204 . 5425

[12] R.G. Edwards, B. Joó (SciDAC Collaboration, LHPC Collaboration, UKQCD Collaboration), Nucl.Phys.Proc.Suppl. 140, 832 (2005), hep-lat/0409003

[13] A. Rothkopf, PoS LATTICE2012, 100 (2012), 1208. 5162

[14] A. Rothkopf, J. Comput. Phys. 238, 106 (2013), 1110.6285

[15] H. Ding, A. Francis, O. Kaczmarek, F. Karsch, H. Satz et al., Phys.Rev. D86, 014509 (2012), 1204.4945

[16] G. Moir, M. Peardon, S.M. Ryan, C.E. Thomas, L. Liu, JHEP 05, 021 (2013), 1301 . 7670

[17] Y. Burnier, A. Rothkopf, Phys.Rev.Lett. 111, 182003 (2013), 1307.6106

The figures and tables must be before references. 